\title{
Jack Goody, La logique de l'écriture
}

\section{Thibault De Meyer}

\section{(2) OpenEdition \\ Journals}

\section{Édition électronique}

URL : http://journals.openedition.org/lectures/24819

ISSN : 2116-5289

Éditeur

Centre Max Weber

Référence électronique

Thibault De Meyer, « Jack Goody, La logique de l'écriture », Lectures [En ligne], Les comptes rendus, 2018, mis en ligne le 11 juin 2018, consulté le 11 juin 2018. URL : http://journals.openedition.org/ lectures/24819

Ce document a été généré automatiquement le 11 juin 2018.

(c) Lectures - Toute reproduction interdite sans autorisation explicite de la rédaction / Any replication is submitted to the authorization of the editors 


\title{
Jack Goody, La logique de l'écriture
}

\author{
Thibault De Meyer
}

1 La logique de l'écriture reprend une série de conférences que Jack Goody (1919-2015) a prononcées à l'université de Chicago et au Collège de France en 1984 et 1985. Le livre a été publié en anglais en 1986, et sa traduction française est parue la même année chez Armand Colin. La présente réédition, chez le même éditeur, conserve la traduction d'Anne-Marie Roussel, mais ajoute une préface d'Éric Dagiral et Olivier Morin.

Bien que Goody, professeur à Cambridge, ait traité de nombreux sujets tels l'évolution de la parenté, le développement des cuisines nationales, l'Islam en Europe... ${ }^{1}$, il reste surtout connu pour ses travaux décrivant les effets de l'écriture sur la cognition et sur l'évolution des sociétés humaines. Comme l'expliquent les préfaciers, la question de l'impact de l'écriture a émergé lors de la Seconde Guerre mondiale, durant laquelle le jeune étudiant en littérature anglaise fut capturé. Alors qu'en Angleterre il était habitué à lire et à écrire quotidiennement, il s'est trouvé privé de papier et de stylo durant l'année où il a été incarcéré dans une prison italienne. Cette expérience l'a conduit à réfléchir sur les effets individuels de l'empêchement d'écrire, mais aussi sur les conséquences que l'absence d'écriture pourrait comporter pour les groupes sociaux.

Le livre le plus cité de Goody est La raison graphique, paru en anglais en $1977^{2}$. Ce livre, qui s'inscrit dans la continuation d'un article important intitulé "The consequences of literacy " écrit avec Ian Watt en $1963^{3}$, se focalise en particulier sur les impacts de l'écriture et des représentations graphiques (telles les listes et les tableaux) sur les compétences cognitives. Il défend notamment l'idée que des contradictions apparaissent beaucoup plus facilement à l'écrit qu'à l'oral, ce qui a pu participer à l'émergence et à la diffusion d'une logique bivalente, qu'on trouve déjà chez Aristote. Dans La logique de l'écriture, Goody vise à imaginer plus généralement quels ont été les impacts de l'écriture sur la société. Pour cela, il se demande successivement comment l'écriture a influencé la religion, l'économie, l'administration étatique et le droit, consacrant un chapitre à chacun de ces domaines.

Concernant les liens encre écriture et religion, Jack Goody défend deux idées centrales. La première est qu'en passant à l'écrit, les préceptes religieux tendent à devenir plus universels. En effet, si les préceptes oraux pouvaient être modifiés pour s'adapter à 
chaque situation d'énonciation, les préceptes écrits sont appelés à s'appliquer de façon univoque dans des contextes hétérogènes. Ainsi, un précepte particulier comme « tu ne tueras point de Juifs ", énoncé oralement dans une situation historique donnée, tend à devenir à l'écrit le précepte général « tu ne tueras point ». L'anthropologue estime qu'en stabilisant les énoncés, l'écriture rend le contexte d'énonciation moins important. En outre, la fixité de l'écrit impose une permanence des préceptes dans la durée et radicalise les conditions qui permettent de les transformer : alors que, dans la tradition orale, une évolution graduelle, presque imperceptible des préceptes peut s'opérer au fil des générations, seule une révolution peut faire rupture à la transmission d'une tradition écrite. On comprend ainsi l'importance des épisodes révolutionnaires, au cours desquels des textes sont publiquement jetés au feu et des monuments sont incendiés

Dans le deuxième chapitre, Goody examine le lien entre l'écriture et l'économie, qui est probablement le premier champ d'activité où est apparue l'écriture. À travers de très nombreux exemples tirés de l'Égypte ancienne, de l'Assyrie, de la Chine et du Ghana, il montre que l'écrit facilite l'enregistrement des achats, des ventes mais aussi des dettes, en suppléant la mémoire humaine pour retenir de grands nombres de données. Dès lors qu'un créancier consigne par écrit les noms de ses débiteurs, il peut en augmenter le nombre. De même pour les transactions marchandes. L'écriture aurait donc eu un impact important à l'échelle individuelle du marchand mais aussi à l'échelle globale de la société. L'écriture a aussi transformé la manière de régler les litiges, des documents écrits étant pris pour référence. L'écrit a encore permis l'établissement de contrats sur plusieurs générations, avec pour effet de reporter sur les enfants les dettes contractées par un père décédé.

6 L'écriture a également eu un impact important sur l'administration des États, notamment par la constitution de comptes centralisés des taxes et le recensement de la population pour prélever les impôts. Dans le chapitre concernant ces questions, l'anthropologue dialogue en particulier avec Max Weber qui, selon lui, ne mettait pas assez l'accent sur les techniques qui permettent aux États de devenir « rationnels ». L'écriture aurait encore permis d'augmenter les contacts entre États voisins; elle aurait donc eu une place importante dans les relations interétatiques, ce qui n'est pas souvent relevé dans les études sur l'écriture. Enfin, Goody souligne les changements qu'a pu subir le droit sous l'influence de l'écriture. Il commence le dernier chapitre en expliquant qu'avant l'instauration d'un code écrit, il n'y a pas de distinction claire entre la coutume et le droit : était réputé permis tout ce qui se pratiquait habituellement. L'écrit a eu pour effet de séparer catégoriquement ce qui est permis de ce qui se fait d'habitude. Le premier correspond au de jure, le texte de loi, alors que le second coïncide avec le de facto. Par ailleurs, comme l'anthropologue le remarquait déjà par rapport aux religions, il est plus difficile de modifier une loi stabilisée par écrit qu'une coutume orale qui peut, de génération en génération, évoluer de manière imperceptible. La conscience du changement des lois augmente donc dans une société utilisant l'écriture.

7 Le livre dans son ensemble permet de saisir les multiples implications individuelles et collectives induites par l'usage de l'écriture dans les sociétés humaines. Les exemples traités viennent de contextes très divers: la Mésopotamie, l'Égypte ancienne, l'Europe médiévale, l'Afrique de l'Ouest... Cependant, les comparaisons que Goody propose ne permettent pas d'isoler le facteur de l'écriture d'un ensemble d'autres facteurs tels la présence de l'école, de moyens de production, de moyens de transport... Comme l'ont remarqué certains critiques, l'écriture n'est jamais une cause suffisante pour entrainer les 
effets décrits par Goody ${ }^{4}$. Ainsi, il existe des religions qui utilisent l'écriture mais qui ne visent pas l'universalisme; Goody lui-même cite le taoïsme en Chine, par exemple (p. 231). L'écriture n'est pas non plus une cause nécessaire aux dynamiques décrites par l'auteur. Goody mentionne le cas des Incas qui se sont constitués en empire sans écriture (p.127). Avec Leibniz, on pourrait cependant comprendre l'écriture comme une cause inclinante $^{5}$ : ce que montre Goody, c'est qu'elle instaure des tendances et que les développements qu'elle suscite "font leur apparition sur le long terme» (p. 72) : ils ne sont donc ni immédiats ni inéluctables.

Dans La logique de l'écriture, Jack Goody explore plusieurs domaines où l'écriture a pu jouer un rôle important dans le développement des sociétés humaines. Il invite par ailleurs les anthropologues à étudier dans des contextes plus précis la manière dont l'écriture s'insère dans la société et la transforme. Notons que si lui-même n'a jamais entrepris un tel projet, Goody encourage également à étudier les effets d'autres technologies de communication telles celles des ordinateurs (p. 33). Dans cette réédition, la traduction initiale du sous-titre de l'ouvrage (Aux origines des sociétés humaines) a été révisée dans un souci de plus grande fidélité à la version anglaise ainsi qu'au contenu du livre : L'écrit et l'organisation de la société. Cette modification nous semble heureuse. On pourrait même se demander si ce sous-titre n'aurait pas pu prendre la place du titre, qui induit quelque peu en erreur puisque dans ce livre, il n'est pas question de «logique » à proprement parler (Goody aborde ce thème dans d'autres travaux, notamment dans La raison graphique susmentionné). Cette réédition aurait gagné à bénéficier d'une meilleure relecture pour corriger quelques coquilles. En outre, l'ajout d'un index aurait été bienvenu pour faciliter les recherches à travers les nombreux éléments historiques et ethnographiques qui nourrissent le livre.

\section{NOTES}

1. Sur ces différents pans de la pensée de Goody, voir respectivement : L'évolution de la famille et du mariage en Europe, Paris, Armand Colin, 1985 ; Cuisines, cuisine et classes, Paris, Centre Georges Pompidou, 1985 ; L'islam en Europe, Paris, La Découverte, 2006.

2. Goody, La raison graphique. La domestication de la pensée sauvage, Paris, Minuit, 1979 (1977 édition originale).

3. Jack Goody \& Ian Watt, "The Consequences of Literacy ", Comparative Studies in Society and History, $\mathrm{n}^{\circ}$ 5, 1963, p. 304-345.

4. Pour une critique, voir notamment John Halverson, «Goody and the Implosion of the Literacy Thesis ", Man, n² 27, 1992, p. 301-317.

5. Pour ce concept leibnizien, voir par exemple : Leibniz, Die philosophischen Schriften von Gottfried Wilhelm Leibniz, vol. 7, Berlin, Weidmann, 1890, p. 110. 


\section{AUTEUR}

THIBAULT DE MEYER

Doctorant au département de philosophie à l'Université de Liège. 